\title{
EOSINOPHILIC FASCIITIS - A DIAGNOSIS TO CONSIDER. CASE REPORT AND REVIEW OF THE LITERATURE
}

\author{
Marina S., Kr. Semkova \\ Department of Dermatology and Venereology, Medical University - Sofia
}

Reviewed by: Prof. R. Madjov

\begin{abstract}
Eosinophilic fasciitis is a rare sclerodermatous disorder with controversial etiology and pathogenesis. Constellation of clinical features and laboratory abnormalities establishes the diagnosis: deep induration of the subcutaneous tissue, flexion joint contractures, high eosinophil count in blood and bone marrow, characteristic histologic findings, arthralgia, myalgia, negative visceral involvement, negative Raynaud phenomenon, history of excessive physical exercise, therapeutic sensitivity to corticosteroids and characteristic MRI findings. The mainstay of treatment are systemic corticosteroids and early intervention is usually effective with a benign course of the disease. Refractory cases may require immunosuppressive or alternative agents.
\end{abstract}

Key words: fasciitis, eosinophils, MRI, corticosteroids

\section{INTRODUCTION}

Eosinophilic fasciitis (EF) is an uncommon connective tissue disease with sclerodermic skin changes, inflammation of the muscle fascia, peripheral eosinophilia, hypergammaglobulinemia and elevated erythrocyte sedimentation rate. Initially described by Shulman in 1974(1), the disease still remains controversial regarding the etiology, pathogenesis and its distinctive nature. Currently, over 250 patients have been reported in the literature, but large case series are few and the understanding of main aspects of this disorder is yet to be elucidated (2). This review discusses the clinical, pathological and laboratory implications of EF, as well as the importance to consider this disease among the differential diagnoses of the more common, clinically similar dermatological and rheumatologic entities.

\section{Case report}

A 69-year old woman presented to our clinic because of a failure of her erysipelas treatment. Three days after excessive physical exercise she felt a strong pain in her right elbow with malaise and fever. Two days later, erythema and oedema occurred first over the elbow region and later on progressed to involve the whole distal part of the arm. The patient felt stiffness of the elbow joint. Treatment for erysipelas with antibiotics, antipyretics and antiseptics was started with a poor outcome. Physical examination revealed unilateral induration of the affected skin with a peau d'orange appear-

\section{Address for correspondence:}

Kr. Semkova, Dept. of Dermatology and Venereology

Medical University, 1, G. Sofiiski Str. Sofia 1431

e-mail: kristina semkova@yahoo.com ance and total immobilization of the elbow joint (Fig.1, Fig.2). The other body parts were spared and the patient was otherwise well. A full-thicknes incisional biopsy specimen showed sclerotic reticular dermis with a focal infiltration with lymphocytes, plasma cells and histiocytes; the underlying fascia was thickened (Fig.3). The blood eosinophils

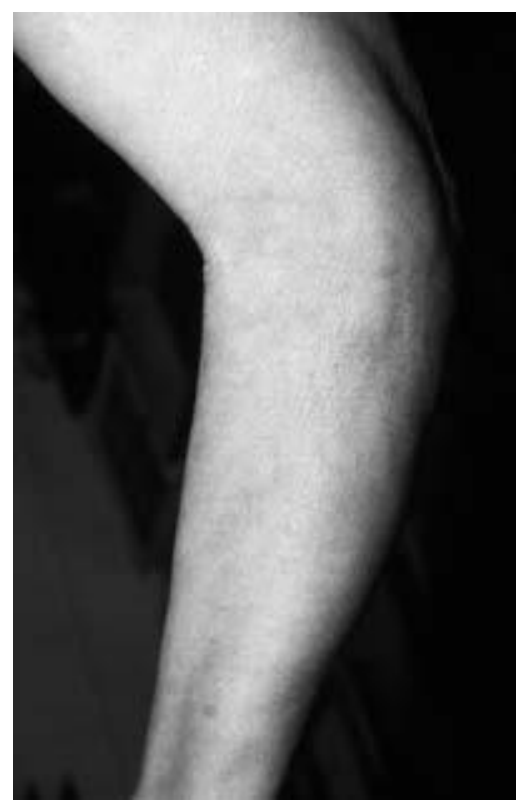

Fig. 1. Induration of the left arm with contracture of the elbow joint.

count and the erythocyte sedimentation rate were elevated. Based on these clinical and laboratory findings, the diagnosis of EF was made, and Methylprednisolon 60mg/daily 
was started. Clinical improvement occurred rapidly and the changes resolved fully in the course of two months.

\section{Etiology and pathogenesis}

The etiology of EF remains obscure and many factors have been proposed so far with supporting evidence of a variable consistency. In half of the cases the onset of the disease is triggered by intense physical exercise or trauma $(3,4)$. Multiple drugs and chemical agents, including L-tryptophan (4), phenytoin (5), simvastatin (6) and trichloroethylene (7), have also been implicated in the pathogenesis of EF. Causal association has been proposed for Borrelia based on the positive serology in several patients, but recent studies of Borrelia-specific DNA in the lesions failed to prove a connection (8). The authors suggested that the Borrelia-positive serology presents an epiphenomenon resulting from the high level of infection in the endemic areas.

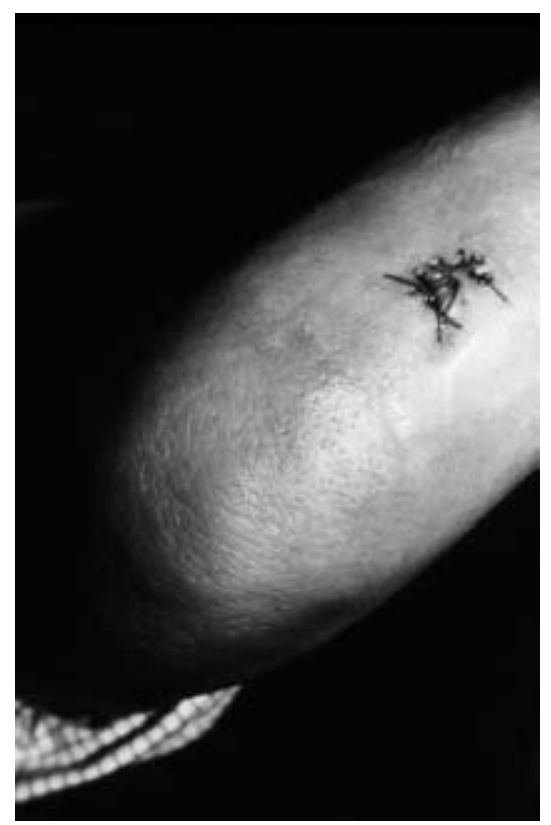

Fig. 2. Induration of the skin below the elbow with a peau d'orange appearance. The stitches indicate the site of the biopsy.

The etiologic factor initiates an inflammatory response in the affected tissues with recruitment of inflammatory cells and release of cytokines stimulating fibroblasts to synthesize collagen and other matrix proteins. Increased levels of IL-5, a potent activator of mature eosinophils, interferon-gamma, activator of tissue macrophages and T-cells, and TGF-beta, a factor stimulating fibroblasts for excessive production of procollagen, have been detected in patients with $\mathrm{EF}(9,4)$. In situ hybridization showed that fibroblasts from lesional skin express in excess type 1 collagen mRNA and TGF-beta in the fascia of patients with EF (10).

\section{Clinical features}

EF may occur at any age, but is most common between the $3^{\text {rd }}$ and $6^{\text {th }}$ decade. Both sexes are equally affected $(3,11)$. Slight racial predilection towards Caucasians is observed.
The disease may start with prodromal symptoms, such as malaise, arthralgia, myalgia, fever. These symptoms precede with weeks to months the cutaneous manifestation or develop concomitantly (11).

Table 1. Diagnostic criteria for eosinophilic fasciitis (11)

\begin{tabular}{||l||}
\hline \multicolumn{1}{|c||}{ Diagnostic criteria for $\mathbf{E F}$} \\
\hline $\begin{array}{l}\text { Deep induration of the subcutaneous tissue, sparing face and } \\
\text { distal extremities }\end{array}$ \\
\hline Flexion joint contractures \\
\hline High eosinophil count in blood and bone marrow \\
\hline Increased ESR and gammaglobulins \\
\hline Characteristic histological findings \\
\hline Myalgia, arthralgia \\
\hline Negative visceral involvement; negative Raynaud phenomenon \\
\hline Initiation after excessive physical activity \\
\hline Corticosteroids sensitive \\
\hline Characteristic MRI findings \\
\hline \hline
\end{tabular}

\section{Cutaneous manifestations $(4,3)$}

Cutaneous lesions develop in three stages in the course of one to several days. Patients first present with rapid onset of erythematous swelling and nonpitting oedema that evolve into peau d'orange or cobblestone appearance with venous furrowing. The final stage is characterized by woody induration and fibrosis. Other cutaneous changes that may rarely occur include urticaria, bullae, alopecia, hypo- and hyperigmentation. Upper and lower extremities are most often involved, but fingers and toes are always spared. The face and the body are very rarely affected.

Symmetrical involvement is the rule but unilateral EF, similar to our patient, has also been reported (12).

\section{Extracutaneous manifestations $(4,3)$}

The most common extracutaneous finding is joint stiffness that progresses to joint contractures secondary to induration and fibrosis of the subcutaneous tissue $(50-75 \%$ of patients). Wrist, elbow and knee joints are most commonly affected. Arthritis (in roughly $40 \%$ of the patients) and carpal tunnel syndrome (16-23\% of the patients) can either precede or follow the skin findings.

Reports of visceral involvement are very limited and visceral symptoms warrant consideration of an alternative diagnosis. Isolated cases of pericarditis, pulmonary, gastrointestinal involvement and peripheral neuropathy have been reported (4).

\section{Associated diseases}

$\mathrm{EF}$ is mainly associated with haematologic disorders, namely aplastic anaemia, thrombocytopenia, chronic lymphocytic leukemia and myelomonocytic leukemia, thrombocytopenic purpura, pancytopenia $(3,4,11)$. The co- 
existence of $\mathrm{EF}$ with hematologic and non-hematologic malignancies, such as breast cancer (13), colorectal carcinoma (14) and T-cell lymphomas, may represent a paraneoplastic phenomenon, but the available information is limited to allow for clear cut screening recommendations.

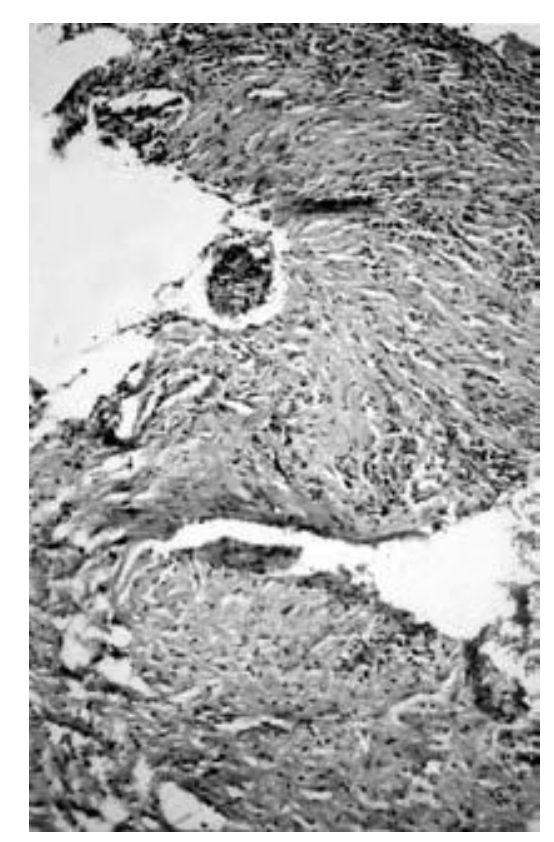

Fig. 3. Histological examination. $H \& E$, x10. Sclerotic reticular dermis, focal infiltration with lymphocytes, plasma cells and histiocytes.

\section{EF in children}

The disease may affect children, albeit rarely, with similar clinical, laboratory and therapeutic features. The differencies are that girls are predominantly affected, arthritis is less frequent, there is more pronounced histopathologic involvement of muscles, hematologic associations are lacking and most patients progress to a benign, scleroderma-like cutaneous fibrosis (15).

\section{Laboratory work-up Laboratory findings}

The most characteristic laboratory findings in EF are peripheral eosinophilia ( $63 \%$ of the patients), hypergammaglobulinemia (35\% of the patients) and elevated ESR ( $29 \%$ of the patients) (4). However, peripheral eosinophilia is not indispensable to make a diagnosis of $\mathrm{EF}$ and does not correlate with clinical severity (16). Therapy initiation results in rapid normalization of the test results in most of the patients, but dissociation between the laboratory findings and the clinical course is not uncommon. Other rare laboratory findings include low titer of antinuclear antibodies, rheumatoid factor and inflammatory markers that may be occasionally present, but are of an insignificant importance.

\section{Imaging}

Imaging studies are becoming an indispensable part of the diagnosis of EF, particularly in dubious cases. MRI findings are specific and include fascial thickening, signal hyperintesity and fascial contrast enhancement. Besides its diagnostic value, MRI reflects the clinical activity of the disease and could be used to monitor the treatment response. In relapse cases, MRI is useful in assessing whether a genuine relapse or a new, unrelated condition has occurred (17).

\section{Histopathology}

Full-thickness incisional skin biopsy gives the definitive diagnosis in both typical and atypical cases. The sample should be deep and should include a continuity of the skin, fat, fascia and superficial muscle.

The histologic findings depend on the stage of progression of cutaneous lesions. Early acute stages show oedema and a mixed inflammatory infiltrate of lymphocytes, plasma cells, histiocytes and occasionally eosinophils in the fascia. Eosinophils may not be present but there is a strong correlation with their count in the blood. Later on, generalized sclerosis and thickening of the fascia and the adjacent structures replace the inflammatory changes and the histologic picture presents with thick hyalinezed collagen bundles with small islands of fat cells trapped in between them $(11,3,4)$. Similar changes, but to a mild degree, develop in the connective tissue components of the underlying subcutaneous tissues, the perimysium and the endomysium (11). Vascular cuffing with inflammatory cells may be observed. A comparison study of the histopathologic features of L-tryptophan induced and non-L-tryptophan induced EF showed that subcutaneous-pancutaneous involvement and dermal and septal neural inflammation are characteristic features of the L-tryptophan associated type (4).

\section{Diagnosis and differential diagnosis}

The diagnosis of EF is suspected in a patient who presents with characteristic cutaneous changes and consistent laboratory findings. It should be confirmed with full-thickness incisional biopsy or characteristic MRI findings.

Several authors have proposed standard diagnostic criteria for the clinical diagnosis of EF, but their wide acceptance is limited by the insufficient sample of patients (Table 1.).

Every criterion is more or less relative; however the characteristic histological findings from deep skin biopsy samples and the MRI findings are considered major criteria for diagnosis.

The clinical presentation of EF may well mimic other far more common syndromes and this often precludes a timely diagnosis. This may result in misdiagnosing the patient and, hence, in underreporting and lower prevalence estimates for EF. The localized and systemic forms of scleroderma are the most important entities in the differential diagnosis. Their differentiation form EF is based on the lack of peripheral eosinophilia. Furthermore, systemic sclerosis is characterized by Raynaud phenomenon, teleangiectasias, abnormal capillaroscopy and visceral involvement, findings that are usually absent in EF.

Eosinophilia-myalgia syndrome and toxic oil syndrome are epidemic disorders, caused by ingestion of L-tryptophan and rapeseed oil, respectively. They have similar clinical and histopathological presentation to EF, but are associated 
with serious visceral involvement and grave outcome. Dermal mucinosis and higher aldolase levels with lower phosphokinase levels are other distinguishing features (11). Scleredema Buschke affects the face and the upper part of the body and the process is restricted to the skin. The disease is usually associated with diabetes and infections. Eosinophilic myositis and perimyositis do not involve the fascia (11). Erysipelas is to be considered at the early stages in patients with unilateral involvement.

\section{Treatment}

Spontaneous resolution has been reported (4), but there are not specific features to predict the outcome, which warrants prompt initiation of treatment.

The mainstay of treatment for $\mathrm{EF}$ are the systemic corticosteroids. Approximately $60 \%$ of the patients respond to daily doses of $40-60 \mathrm{mg}$ oral prednisolone with reduction of oedema and induration of the affected skin. The clinical response is better in patients who present early with predominant inflammatory lesions. Several weeks to months of treatment are necessary for a full recovery. Most authors start with 40-60 mg oral prednisolone and taper it gradually as the disease improves. Additional topical therapy may further increase the effect of the systemic therapy and usually comprises madecassol, dimethylsulphoxide, heparoid. When the response to systemic steroids is poor or a steroid-sparing effect is sought, alternative agents may be used either alone, or as adjuvants to the steroid therapy. These include hydroxychloroquine, azathioprine, methotrexate, ciclosporin A, D-penicillamine and histamine 2 antagonists $(11,16)$. These drugs were used with variable success.

Recently, the antitumour necrosis factor alpha, infliximab, was reported to cause a complete clinical and laboratory remission of the inflammatory process after 1 year of treatment (18).

Photochemotherapy, bath-PUVA and UVA1 in combination with isotretinoin and oral prednisone were successfully used in patients with recalcitrant disease $(19,20)$.

Joint contractures require surgical management and early physical therapy to restore and/or maintain mobility.

Treatment in children should be started with prednisone at doses of $2 \mathrm{mg} / \mathrm{kg} / \mathrm{d}$. Tapering should be considered at normalization of the laboratory parameters. D-penicillamine is a safe alternative for steroid-refractory cases (15).

\section{Prognosis}

Proper treatment usually results in total resolution of the clinical manifestations, but quite often induration may persist for many months afterwards. A study of Endo et al. (2) found the clinical variables that predict persistence of the fibrosis - presence of morphealike skin lesions, younger age at onset, truncal involvement, and presence of dermal fibrosclerosis on histopathologic specimen. The relapse rate has not been estimated.

\section{REFERENCE}

1. Shulman LE. Diffuse fasciitis with eosinophilia: a new syndrome? Trans Assoc Am Physicians. 1975; 88: $70-86$.

2. Endo Y, Tamura A, Matsushima Y, Iwasaki T, Hasegawa M, Nagai Y. Eosinophilic fasciitis: report of two cases and a systematic review of the literature dealing with clinical variables that predict outcome. Clin Rheumatol. 2007; 26: 1445-1451.

3. Lakhanpal S, Ginsburg WW, Michet CJ, et a1. Eosinophilic fasciitis: clinical spectrum and therapeutic response in 52 cases. Semin Arthritis Rheum. 1988; 17: 221-231.

4. Helfman T, Falanga V. Eosinophilic fasciitis. Clinics in Dermatology. 1994; 12: 449-455.

5. Buchanan RR, Gordon DA, Muckle TJ, et al. The eosinophilic fasciitis syndrome after phenytoin (dilantin) therapy. J Rheumatol. 1980; 7: 733-736.

6. Choquet-Kastylevsky G, Kanitakis J, Dumas $\mathrm{V}$, et al. Eosinophilic fasciitis and simvastatin. Arch Intern Med. 2001; 161: 1456-1457.

7. Hayashi N, Igarashi A, Matsuyama T, Harada $\mathrm{S}$. Eosinophilic fasciitis following exposure to trichloroethylene: successful treatment with cyclosporin. Br J Dermatol. 2000; 142: 830-832.

8. Anton, E. Failure to demonstrate Borrelia burgdorferi-specific DNA in lesions of eosinophilic fasciitis. Histopathology. 2006; 49: 88-90.

9. Viallard JF, Taupin JL, Ranchin V, et al. Analysis of leukemia inhibitory factor, type 1 and type 2 cytokine production in patients with eosinophilic fasciitis. J Rheumatol. 2001; 28: 75-80.

10. Peltonen J, Kahari L, Jaakkola S, et al. Evaluation of transforming growth factor beta and type I procollagen gene expression in fibrotic skin diseases by in situ hybridization. $J$ Invest Dermatol. 1990; 94: 365-371.

11. Marina S, Savova Y, Broshtilova V, Kazandjieva J. Fasciitis diffusa cum eosinophilia (Shulman syndrome). Rheumatology. 2005; 1: 7-11.

12. Daniel RS, Brown AN. Case report of unilateral eosinophilic fasciitis in a Vietnamese woman. Am J Med Sci. 2009; 337: 153-154.

13. Watts RA, Merry P. Familial eosinophilic fasciitis and breast cancer. Br J Rheumatol. 1994; 33: 93-94.

14. Philpott H, Hissaria P, Warren L, et al. Eosinophilic fasciitis as a paraneoplastic phenomenon associated with metastatic colorectal carcinoma. Australasian J Dermatol. 2008; 49: 27-29.

15. Grisanti MW, Moore TL, Osborn TG, Harber PL. Eosinophilic fasciitis in children. Semin Arthritis Rheum. 1989; 19: 151-157.

16. Bischoff L, Derk CT. Eosinophilic fasciitis: demographics, disease pattern and response to treatment: report of 12 cases and review of the literature. Int J Dermatol. 2008; 47: 29-35.

17. Moulton SJ, Kransdorf MJ, Ginsburg WW, et al. Eosinophilic fasciitis: spectrum of MRI findings. AJR. 2005, Vol. 184, pp. 975-978.

18. Tzaribachev N, Holzer U, Schedel J, et al. Infliximab effective in steroid-dependent juvenile eosinophilic fasciitis. Rheumatology. 2008; 47: 930-932. 
19. Weber HO, Schaller M, Metzler G, et al. Eosinophilic fasciitis and combined UVA1-Retinoid-Corticosteroid treatment: two case reports. Acta Derm Venereol. 2008; 304-306.
20. Schiener R, Behrens-Williams SC, Gottlober $\mathrm{P}$, et al. Eosinophilic fasciitis treated with psoralen-ultraviolet A bath chemotherapy. $\mathrm{Br} J$ Dermatol. 2000; 142: 804-807. 